\title{
Phase separation in mixtures of a rodlike colloid and two or more rodlike polymers
}

\author{
Richard P. Sear ${ }^{\text {a) }}$ and Daan Frenkel ${ }^{\text {b) }}$ \\ FOM Institute for Atomic and Molecular Physics, Kruislaan 407, NL-1098 SJ Amsterdam, The Netherlands
}

(Received 5 July 1996; accepted 5 September 1996)

\begin{abstract}
A suspension of rodlike colloidal particles and rodlike liquid crystalline polymers is modelled as a mixture of thick (colloidal) and thin (polymeric) hard rods. Extensive immiscibility in the fluid phase is observed in the mixtures. For two species of polymers, one species much longer than the other, we observe two demixing critical points. For three polymer species we find three critical points. Polymer molecules of length $l$ induce an attractive interaction of range $l$ between colloidal rods. Two different polymers induce effective attractions of two different ranges. The range of the attraction determines the density at which the demixing occurs. The attractions of different ranges create demixing at different densities. (c) 1996 American Institute of Physics.
\end{abstract} [S0021-9606(96)51146-3]

\section{INTRODUCTION}

Mixtures of hard convex particles commonly show immiscibility. In particular, mixtures of hard rods of different diameters demix in both the isotropic and the nematic phases. ${ }^{1-3}$ Earlier work by one of us and Jackson ${ }^{1}$ has shown that a mixture of rods of finite diameter with rods of zero diameter shows extensive immiscibility in the isotropic fluid phase. The mixture is an idealized model of a mixture of synthetic colloidal rods ${ }^{4}$ and a rigid rodlike polymer. ${ }^{5}$ Here we generalize the theory of Ref. 1 to two or more polymer species and consider the phase behavior of the resulting many-component mixtures. Given that polymers can be prepared with a range of molecular weights this situation has clear relevance. The different rodlike polymer species are of different lengths.

We find that if the lengths of the polymer species are very different, the mixture's phase diagram is almost a superposition of the phase diagrams of the binary mixtures formed from the colloid and one of the polymers. The ternary mixture of colloid plus two polymer species then possesses two demixing critical points, each at almost the same position as the critical point of a binary mixture. The two critical points are at the ends of two coexistence curves, these two curves meet at a triple point where three fluid phases coexist. These findings are reminiscent of those of Stell and Hemmer ${ }^{6,7}$ for a very different model.

In the following section we derive the necessary theory and present representative phase diagrams. As the theory is simply a generalisation of that given in Ref. 1, it is not given in any detail (see Ref. 1 for a more thorough discussion). We discuss our results from a perspective in which all components are considered explicitly and from a perspective in which only the colloid is considered explicitly but in which the colloid-colloid interaction is modified by the presence of polymer. ${ }^{8}$ We conclude with a summary and brief comparison with other work.

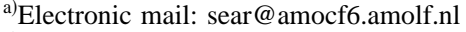

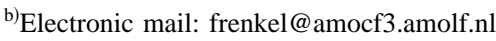

\section{THEORY}

The model mixtures are composed of one colloidal component and $n$ polymer components. The colloid is a spherocylinder of length $L$ and diameter $D$ (Ref. 5) and the $i$ th polymer is a rod of length $l_{i} L$ and diameter $0,{ }^{9}$ see Fig. 1 . We will consider only the case in which all $l_{i} \gg 1$. We will consider only isotropic phases and so our rodlike polymers need not be very rigid; their persistence length could be of the same order as their length $l_{i} L$. In the isotropic phase the colloid-polymer interaction depends only weakly on the persistence length of the polymer, ${ }^{5}$ just as the interaction between semi-flexible polymers depends only weakly on their persistence length. ${ }^{5,10}$

The theoretical expressions for the thermodynamic functions are straightforward generalizations of those in Ref. 1. The free energy $A$ for a mixture of one colloid and $n$ polymers, is

$$
\begin{aligned}
\frac{\beta A\left(N_{c}, \mathbf{N}_{p}, V\right)}{V}= & \rho_{c}\left[\ln \rho_{c} \Lambda_{c}-1\right]+\sum_{i=1, n} \rho_{p, i}\left[\ln \rho_{p, i} \Lambda_{p, i}-1\right] \\
& +\rho_{c}^{2} v_{0}+\rho_{c} \sum_{i=1, n} \rho_{p, i} l_{i} v_{0}
\end{aligned}
$$

where $\rho_{c}=N_{c} / V$ and $\rho_{p, i}=N_{p, i} / V$ are the number densities of the colloid and of the polymers, respectively. The vector $\mathbf{N}_{p}$ represents the set of all $N_{p, i}$. The leading term of the second virial coefficient of a fluid of the colloidal rods is $v_{0}=(\pi / 4) L^{2} D . \Lambda_{c}$ and $\Lambda_{p, i}$ are the thermal volumes of the colloid and of the polymers, respectively. There are no $\rho_{p, i} \rho_{p, j}$ terms in Eq. (1) because the diameters of the polymeric rods are 0 ; thus the volume excluded to one polymeric rod by another is zero. However, even though the polymer diameter is zero, a colloidal rod excludes a polymeric rod from the finite volume $l_{i} v_{0}$. Eq. (1) becomes exact in the limit that the lengths of the colloid and of all the polymers are much greater than the diameter of the colloid. ${ }^{5}$

The chemical potentials $\mu_{p, i}$ of the polymers are

$$
\beta \mu_{p, i}\left(\rho_{c}, \rho_{p, i}\right)=\ln z_{p, i} \Lambda_{p, i}=\ln \rho_{p, i} \Lambda_{p, i}+\rho_{c} l_{i} v_{0},
$$




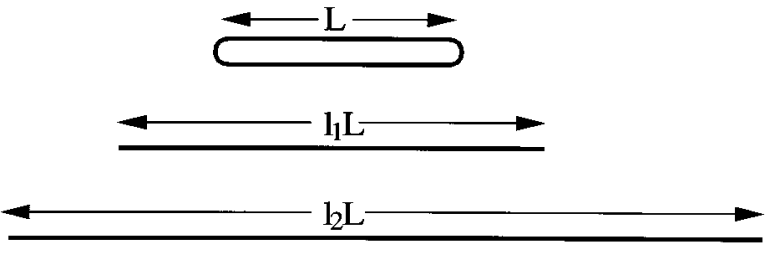

FIG. 1. The models for the colloid (top) and two polymer species (middle and bottom). The diameter of the colloidal spherocylinder is $D$.

where the first equality defines the activity of the $i$ th polymer species $z_{p, i}$. It is more convenient to work with the semigrand potential $\Omega$ than with the free energy. $\Omega$ is a Legendre transform ${ }^{11}$ of the free energy

$$
\Omega=A-\sum_{i=1, n} N_{p, i} \mu_{p, i} .
$$

Substituting Eqs. (1) and (2) in (3) we obtain

$$
\begin{aligned}
\frac{\beta \Omega\left(\rho_{c}, \mathbf{z}_{p}, V\right)}{N_{c}}= & \ln \rho_{c} \Lambda_{c}-1+\rho_{c} v_{0} \\
& -\sum_{i=1, n} \frac{z_{p, i}}{\rho_{c}} \exp \left(-\rho_{c} l_{i} v_{0}\right) .
\end{aligned}
$$

We now neglect the colloid-colloid interactions, i.e., we take the limit of all $l_{i}$ much larger than 1 . This reduces the number of parameters required to specify a mixture by 1 and removes most of the driving force for a transition to a nematic phase. Because we now have interactions only between rods of different species the excluded volume interactions can be reduced by demixing, without having to form a nematic phase. Indeed, without the colloid-colloid interactions and for only one polymer species our mixture corresponds to the so-called Widom-Rowlinson mixture, ${ }^{1,2,8,12,13}$ treated at the mean-field level. This mixture of two rods with only an interaction between rods of different species almost certainly does not undergo a transition to the nematic phase. ${ }^{1,13}$ It seems likely that increasing the number of polymer species does not change this picture, there is probably still no nematic phase. Note that the size of the colloid-polymer interaction term in Eq. (4) decreases exponentially with increasing colloid density. As the formation of a nematic phase will be driven by this term it is difficult to see how a stable nematic phase can be formed. With no nematic phase present we can be sure that our phase diagrams will be correct, the demixing cannot be metastable with respect to an isotropic-nematic transition.

So, we neglect the third term of Eq. (4) and define reduced variables

$$
\begin{aligned}
& \rho=\rho_{c} v_{0} l_{1}, \quad \rho_{i}=\rho_{p, i} v_{0} l_{1}, \quad z_{i}=z_{p, i} v_{0} l_{1}, \\
& \lambda=v_{0} l_{1} \Lambda_{c}^{-1} \exp \left(\beta \mu_{c}\right),
\end{aligned}
$$

where $\mu_{c}$ is the chemical potential of the colloid. We have reduced the densities by the second virial coefficient of the interaction between the colloid and polymer species 1 . The

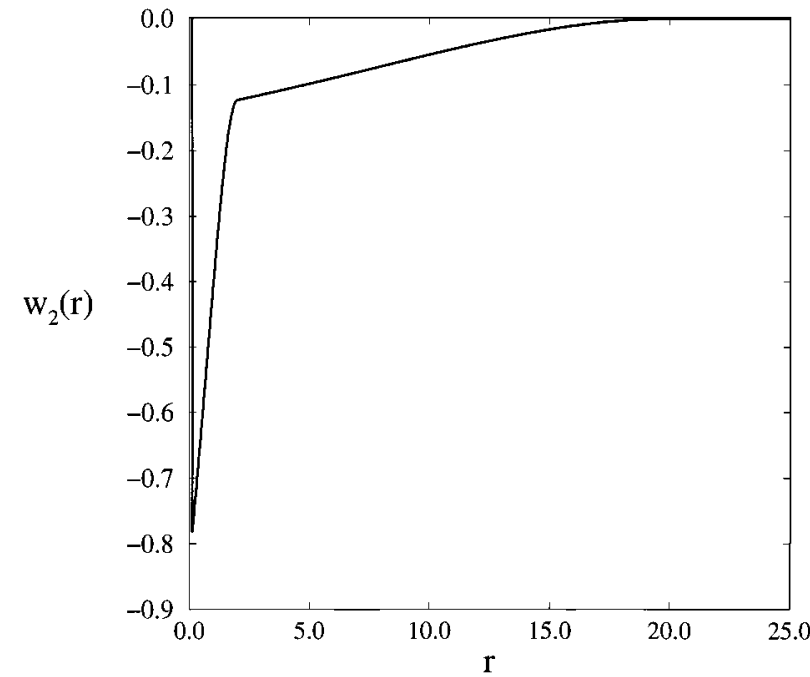

FIG. 2. The low density limit of the potential of mean force $w_{2}$ as a function of separation $r$ (Ref. 14). The system is not rods but spheres, due to the complexity of orientationally averaging the interaction between rods. The potential of mean force is that between two "colloidal" spheres which interact via a hard-sphere potential of range 0.1 . Each colloidal sphere interacts with "polymer" spheres of species 1 and 2 via hard-sphere interactions of diameters 1 and 10, respectively. The reduced activities of "polymer" species 1 and 2 are 1 and 0.2 , respectively.

calculation of phase coexistence requires the pressure $p$ and the chemical potential of the colloid. In reduced units, they are

$$
\beta p v_{0} l_{1}=\rho+\sum_{i=1, n} z_{i}\left(1+\rho \frac{l_{i}}{l_{1}}\right) \exp \left(-\rho l_{i} / l_{1}\right)
$$

and

$$
\ln \lambda=\ln \rho+\sum_{i=1, n} z_{i} \frac{l_{i}}{l_{1}} \exp \left(-\rho l_{i} / l_{1}\right) .
$$

\section{DEMIXING}

A mixture of a colloid and one polymer species was studied in Ref. 1. Extensive immiscibility in the isotropic phase was observed, see Fig. 2 of Ref. 1. For this mixture, it was shown that the critical colloid density $\rho^{*}$ and polymer activity $z^{*}$ are ${ }^{1}$

$$
\rho^{*}=\frac{l_{1}}{l_{i}}, \quad z_{i}^{*}=e \frac{l_{1}}{l_{i}} .
$$

Recall that we have assumed all $l_{i} \gg 1$. Both $\rho^{*}$, and $z^{*}$ are inversely proportional to $l_{i}$. The colloid density at phase separation is inversely proportional to $l_{i}$ because $l_{i}$ determines the range of the indirect interaction between pairs of colloidal rods due to the polymer. Viewed from the perspective of the colloid, ${ }^{8,12,14}$ the polymer induces an attraction between two colloidal rods which are less than $l_{i} L$ apart. Each colloidal rod excludes polymer of type $i$ from volume $v_{0} l_{i}$ surrounding it. ${ }^{5}$ When two colloidal rods are less than $l_{i} L$ apart their two excluded volumes overlap ${ }^{15-17}$ reducing the total volume excluded to a polymer rod. This increase in 

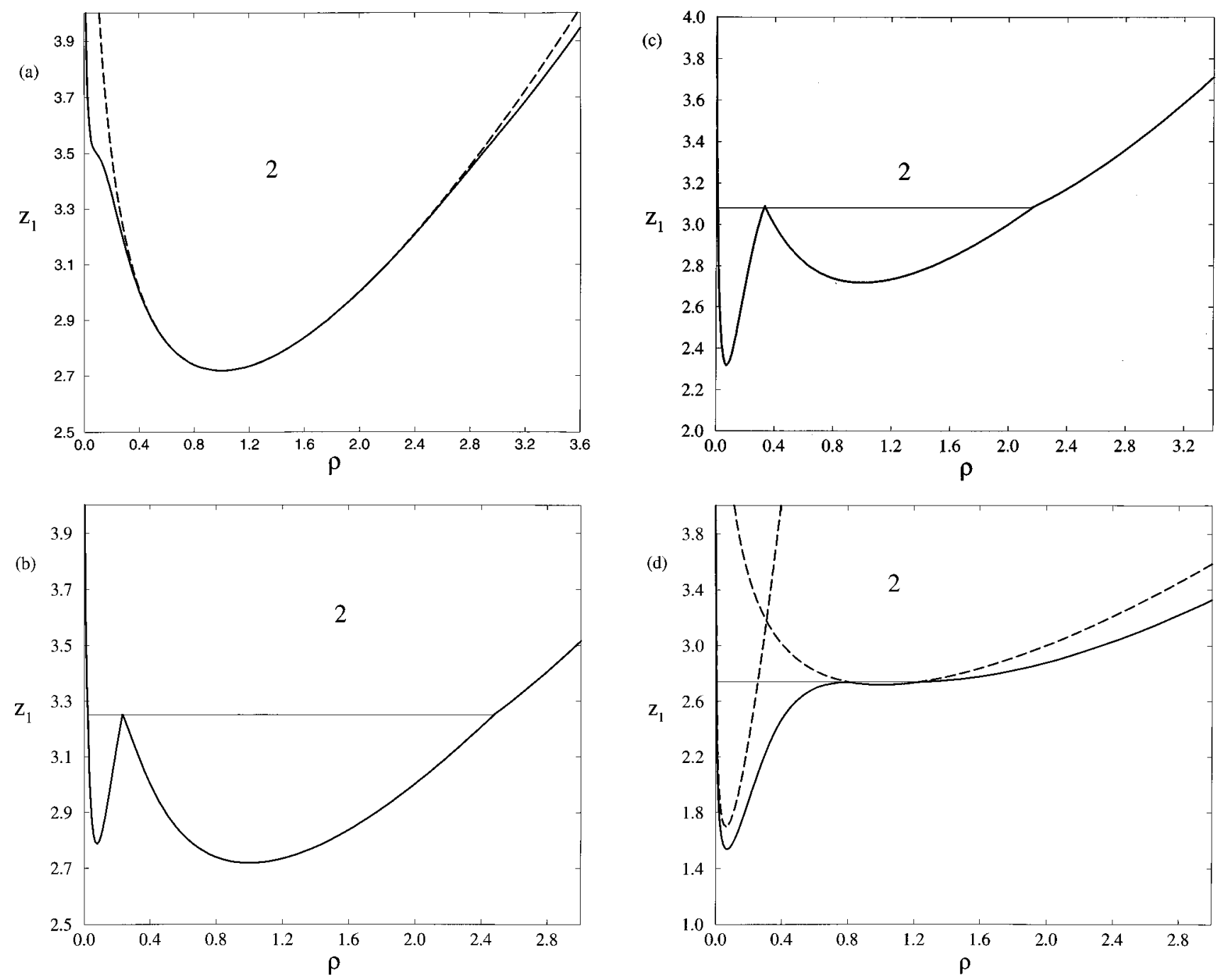

FIG. 3. The phase diagram for a mixture of colloidal rods with two species of polymeric rods, $l_{2} / l_{1}=16$. The thick solid curves separate the one and two phase regions, the thin solid lines connect the triple point densities and the dashed curves separate the one and two phase regions for mixtures of the colloid with either polymer 1 or polymer 2 . The two phase regions are denoted by the number 2 . The phase diagram of an athermal ternary mixture is three dimensional. We show four slices: (a) $z_{2} / z_{1}=0.035$, (b) $z_{2} / z_{1}=0.05$, (c) $z_{2} / z_{1}=0.0625$ and (d) $z_{2} / z_{1}=0.1$.

the volume available to a polymer rod increases the translational entropy of the polymer. The increased translational entropy of the polymer biases configurations in which colloidal rods are close, acting as an attractive potential between them.

Of course, the polymer induced interaction between two colloidal rods depends on their orientations. When the two rods are only a little under $l_{i} L$ apart, for almost all orientations their excluded volumes do not overlap. Thus, the orientationally averaged interaction between two colloidal rods is very weak for separations near $l_{i} L$. It is this rapid decay of the interaction with separation that makes the interaction scale as $l_{i}$ not $l_{i}^{3}$.

The importance of the observation that the interaction range depends on the length of the polymer is that by changing the polymer we can change the range of the effective interaction, the potential of mean force, ${ }^{18}$ between the colloidal rods. The importance of the range of attractive interac- tions between particles is well established. For example, it has been shown that if the interaction range is made very short the vapor-liquid critical point moves to within the solid to become an expanded-solid-condensed-solid critical point. ${ }^{19-21}$ So, the critical density depends on the range of the potential of mean force. What if the interaction is characterized by two (or more) ranges? Such a potential is shown in Fig. 2. This will be the case for the potential of mean force between colloidal rods in the presence of a mixture of polymers of different lengths. If the polymer lengths are similar then the critical density is just some average of the critical densities in the presence of only one of the polymers. However, if the polymers are of widely differing lengths we find multiple critical points. Essentially, we find a critical point at each $\rho^{*} \sim l_{1} / l_{i}$.

For a mixture of a colloid and two polymer species there is only one parameter, the ratio of the lengths of the two polymer rods, $l_{2} / l_{1}$. For $l_{2} / l_{1}=16$ the phase behavior is 
shown in Fig. 3. The two critical points are obvious. Also clear, is that in order to observe two critical points the ratio between the activities of the polymers should be approximately $z_{2} / z_{1} \sim l_{1} / l_{2}$. If this ratio is too large the critical point associated with polymer 1 (i.e., the high density critical point) is within the two phase region associated with polymer 2 and if the ratio is too small the low density critical point is within the two phase region [see Fig. 3(a)].

Note that the high density critical point is at almost exactly the same $\rho$ and $z_{1}$ as for a mixture of the colloid and just polymer 1 . The addition of polymer 2 , although it creates an additional critical point at low densities has very little effect at densities around unity. This is because, at constant activity, the density of a polymer decays exponentially with increasing colloid density, see Eq. (2). Thus, at the high density critical point the density of polymer 2 is $\sim \exp (-16)$, far too small to effect the phase behavior. At the low density critical point although the density of the shorter polymer, polymer 1, is larger than that of polymer 2 it plays little role in inducing demixing because the contribution of polymer 1 to the colloid's chemical potential is almost constant. Two phases with different colloid densities can only coexist if the differences in pressure and colloidal chemical potential induced by the difference in colloidal density are counterbalanced by changes in the polymer density. Consider the equation of the spinodal ${ }^{1}$

$$
\left(\frac{\partial \ln \lambda}{\partial \rho}\right)_{\mathbf{z}_{p}, T}=\rho^{-1}-\sum_{i=1, n} z_{i}\left(\frac{l_{i}}{l_{1}}\right)^{2} \exp \left(-\rho l_{i} / l_{1}\right)=0 .
$$

For two polymer species and for $z_{2} / z_{1}=l_{1} / l_{2}$, Eq. (9) becomes

$$
\rho^{-1}-z_{1} \exp (-\rho)-z_{1} \frac{l_{2}}{l_{1}} \exp \left(-\rho l_{2} / l_{1}\right)=0 .
$$

For $\rho \sim l_{1} / l_{2}$ and $z_{1} \sim 1$ there are two solutions of Eq. (10) in which the third term dominates the second, for $l_{2} \gg l_{1}$. At the two lower densities at the triple point of Fig. 3(b) the densities of polymer 1 are 3.28 and 2.58. Even here, at the highest polymer activity at which there are two distinct coexistence curves, the drop in the density of polymer 1 between the phases with the low and high colloid density is small. In contrast the densities of polymer 2 are $1.16 \times 10^{-1}$ and $4.04 \times 10^{-3}$, a drop of almost 2 orders of magnitude. There are also two solutions of Eq. (10) with $\rho \sim 1$, in which the third term is very small, for $l_{2} \gg l_{1}$. These relate to the higher density coexistence.

We can also describe the appearance of two critical points using the language of colloidal rods interacting via an effective potential, i.e., we treat the mixture as a fluid of colloidal rods interacting via a potential of mean force. The potential of mean force between colloidal rods has associated with it an effective energy. ${ }^{8}$ We thus have an effective thermodynamic energy and can view demixing as separation into a dilute (gas) phase with high entropy but high energy and a dense (liquid) phase with low entropy and low energy. This is simply the classical van der Waals picture. With a poten-

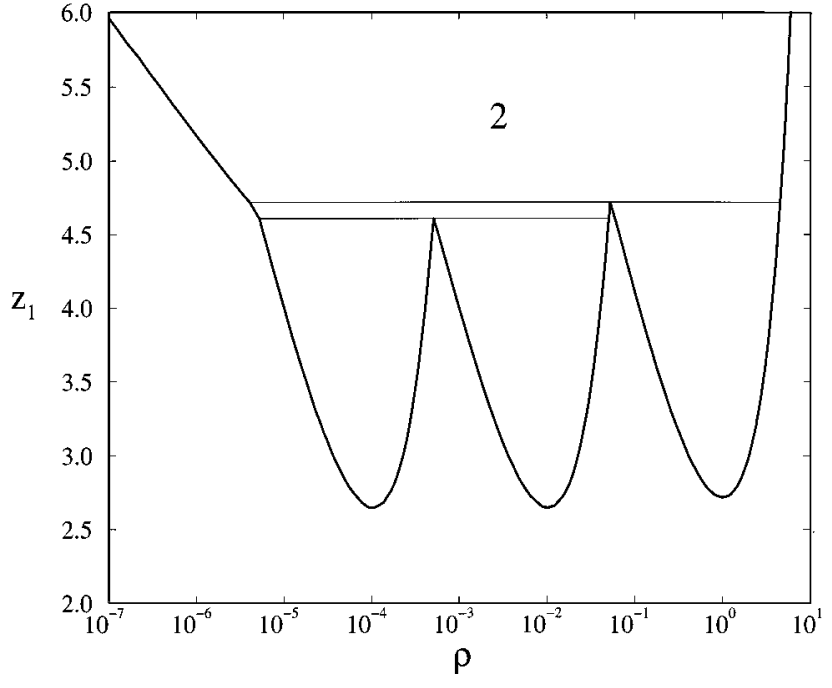

FIG. 4. The phase diagram for a mixture of colloidal rods with three species of polymeric rods, $l_{2} / l_{1}=10^{2}$ and $l_{3} / l_{1}=10^{4}$. The thick solid curves separate the one and two phase regions and the thin solid lines connect the triple point densities. The two phase region is denoted by the number 2 . $z_{2} / z_{1}=10^{-2}$ and $z_{3} / z_{1}=10^{-4}$.

tial of the form of Fig. 2 the energy will decrease in two steps, when $\rho \sim l_{2} / l_{1}$ and $\rho \sim 1 .{ }^{19,20}$ Each decrease creates a two phase coexistence region.

There is no restriction to only two polymer species. For three polymer species the mixture is specified by two parameters, $l_{2} / l_{1}$ and $l_{3} / l_{1}$, and the phase diagram is four dimensional. We show a slice through the phase diagram for $l_{2} / l_{1}=10^{2}$ and $l_{3} / l_{1}=10^{4}$ in Fig. 4 . For these large ratios of the polymer lengths the coexistence curves are almost identical, except for their different density scales. The density scales of the three coexistence curves in Fig. 4 are $l_{1} / l_{3}$, $l_{1} / l_{2}$ and 1 , and are set by the lengths of the polymers. As expected we find three critical points and two triple points. Note that Fig. 3 is for fixed ratios between the three polymer activities. We can tune these ratios and bring the two triple points together to create a quadruple point, i.e., four phases in coexistence.

If the number of polymer species is further increased we can create more and more critical points, and by fine-tuning the activity ratios, coexistence between larger and larger numbers of phases. As the number of polymer species is increased the polymers will interfere with each other: demixing which we consider to be driven by polymer species $i$ will be affected by polymer species which are shorter than $i$. However, we are free to make the differences in length between the polymer species arbitrarily large. Making the difference in length large reduces the interference between different coexistence curves. As the polymer's length is increased the colloid density at the critical point decreases as $l_{i}^{-1}$. The interactions between the colloid and the remaining polymer species are proportional to the colloid density and so they decrease. This decrease also reduces the driving force for ordering. In earlier work on symmetrical mixtures, ${ }^{13}$ the number of phases which could coexist was limited because if the number of species was sufficiently large demixing was 
preempted by solidification. Here, it may be possible to have any (finite) number of critical points and, by fine-tuning the activity ratios, any (finite) number of fluid phases coexisting.

\section{CONCLUSION}

Colloidal suspensions with one polymer present have been extensively studied both experimentally and theoretically. ${ }^{4,17,22}$ The strong dependence of the phase behavior on the size of the polymer has been stressed; ${ }^{1,19,21-23}$ below a certain minimum polymer size there is no demixing in the fluid phase. Varying the size of the polymer allows the effective colloid-colloid interaction to be varied in a way which is impossible for simple substances such as argon. Both polymers and colloidal particles, because they are composed of many molecules, can be made in a range of sizes. This allows the preparation of an infinite number of mixtures, with a consequently almost limitless potential for different effective colloid-colloid interactions. In the preceding sections we have studied some rather extreme mixtures. We have seen that by introducing polymers of very different lengths we can induce effective colloid-colloid interactions of very different ranges. The phase behavior is then rather unusual. We see in Fig. 3 that for a range of polymer activities, on increasing the colloid density we go from a onephase region to a two-phase, back to one-phase, into another two phase region and finally back to one phase.

Stell and Hemmer ${ }^{6}$ studied a van der Waals fluid in one dimension; they introduced steps into the repulsive cores of the rods. They were able to show that the fluid showed $n$ transitions if the core had $n$ steps. The results presented here are analogous to those of Stell and Hemmer although our model is very different. In both Ref. 6 and here the parts of the potential or potential of mean-force with different ranges dominate at different densities producing a series of almost independent phase transitions.

For mixtures of thick and thin hard rods, in the limit that all rod lengths are much greater than the diameter of the thick rod and the thick rod is much shorter than any of the thin rods, the free energy Eq. (1) is exact. ${ }^{5}$ Our results, Figs. 3 and 4, are therefore exact for these mixtures. However, of course, mixtures which have free energies similar to that of Eq. (1) will show similar behavior. For example, virus particles are charged, have diameters which are not negligible and may not have very large length to diameter ratios. ${ }^{24}$
None of these problems really change the phase behavior of virus particles qualitatively ${ }^{5,3}$ and the phase behavior of Fig. 3 is simply a consequence of introducing two length scales into the colloid-colloid potential of mean force. We are therefore confident that our results can be reproduced experimentally.

\section{ACKNOWLEDGMENTS}

It is a pleasure to acknowledge a careful reading of the manuscript by R. Blaak. One of us (R.P.S.) would like to thank The Royal Society for the award of a fellowship and the FOM institute AMOLF for its hospitality. The work of the FOM Institute is part of the research program of FOM and is made possible by financial support from the Netherlands Organization for Scientific Research (NWO).

${ }^{1}$ R. P. Sear and G. Jackson, J. Chem. Phys. 103, 8684 (1995).

${ }^{2}$ R. P. Sear and B. M. Mulder, J. Chem. Phys. 105, 7727 (1996).

${ }^{3}$ R. van Roij and B. M. Mulder, J. Chem. Phys. (to be published).

${ }^{4}$ P. A. Buining, A. P. Philipse, and H. N. W. Lekkerkerker, Langmuir 10, 2106 (1994)

${ }^{5}$ G. J. Vroege and H. N. W. Lekkerkerker, Rep. Prog. Phys. 55, 1241 (1992)

${ }^{6}$ G. Stell and P. C. Hemmer, J. Chem. Phys. 56, 4274 (1972).

${ }^{7}$ J. M. Kincaid, G. Stell, and C. K. Hall, J. Chem. Phys. 65, 2161 (1976).

${ }^{8}$ J. S. Rowlinson, Adv. Chem. Phys. 41, 1 (1980).

${ }^{9}$ P. Bolhuis and D. Frenkel, J. Chem. Phys. 101, 9869 (1994).

${ }^{10}$ T. Odijk, Macromolecules 19, 2313 (1986).

${ }^{11}$ D. Chandler, Introduction to Modern Statistical Mechanics (Oxford University Press, New York, 1987).

${ }^{12}$ B. Widom and J. S. Rowlinson, J. Chem. Phys. 52, 1670 (1970).

${ }^{13}$ R. P. Sear, J. Chem. Phys. 104, 9948 (1996).

${ }^{14}$ M. I. Guerrero, J. S. Rowlinson, and B. L. Sawford, Mol. Phys. 28, 1603 (1974).

${ }^{15}$ S. Asakura and F. Oosawa, J. Chem. Phys. 22, 1255 (1954).

${ }^{16}$ A. Vrij, Pure Appl. Chem. 48, 471 (1976).

${ }^{17}$ P. Warren, S. M. Ilett, and W. C. K. Poon, Phys. Rev. E 52, 5205 (1995).

${ }^{18}$ J.-P. Hansen and I. R. McDonald, Theory of Simple Liquids, 2nd ed. (Academic, London, 1986).

${ }^{19}$ P. Bolhuis, M. Hagen, and D. Frenkel, Phys. Rev. E 50, 4880 (1994).

${ }^{20}$ A. Daanoun, C. F. Tejero, and M. Baus, Phys. Rev. E 50, 2913 (1994).

${ }^{21}$ C. F. Tejero, A. Daanoun, H. N. W. Lekkerkerker, and M. Baus, Phys. Rev. E 51, 558 (1995).

${ }^{22}$ W. C. K. Poon and P. N. Pusey, Observation, Prediction and Simulation of Phase Transitions in Complex Fluids, edited by M. Baus et al. (Kluwer, Dordrecht, 1995).

${ }^{23}$ D. Frenkel, P. Bladon, P. Bolhuis, and M. Hagen, Mol. Simul. 16, 127 (1996).

${ }^{24}$ S. Fraden, Observation, Prediction and Simulation of Phase Transitions in Complex Fluids, edited by M. Baus et al. (Kluwer, Dordrecht, 1995). 